\title{
Cerebral Palsy and Rehabilitative Care: The Role of Home-Based Care and Family-Centered Approach
}

\author{
Sonika Agarwal, ${ }^{1}$ Mark S Scher, ${ }^{2}$ Ann Tilton ${ }^{3}$ \\ ${ }^{1}$ Division of Child Neurology, Children's Hospital of Philadelphia, Perelman School of Medicine at the University of Pennsylvania, \\ Philadelphia, PA, USA; ${ }^{2}$ Rainbow Babies and Children's Hospital/MacDonald Hospital for Women, University Hospitals Cleveland \\ Medical Center, Case Western Reserve University School of Medicine, Cleveland, OH, USA; \\ ${ }^{3}$ Section of Child Neurology, Children's Hospital, New Orleans, LA, USA. \\ Correspondence to: Sonika Agarwal, Assistant Professor of Clinical Neurology and Pediatrics, Division of Child Neurology, \\ Children's Hospital of Philadelphia, Perelman School of Medicine at the University of Pennsylvania, Philadelphia, PA, USA. \\ agarwals2@chop.edu
}

$\mathrm{G}$ oswami, et al. [1] published an open-label randomized control trial to evaluate the efficacy of adjunctive individualized homecentered activity-based therapy in children with spastic diplegic cerebral palsy $(\mathrm{CP})$ at ages 5-12 years [1]. Children in the intervention arm were prescribed parent-supervised home-centered activity-based therapy in addition to institutional physiotherapy.Main outcome measures compared mean changes in the 6-minute-walk test scores at 6 month from baseline between the two groups. Adjunct home-centered activity-based therapy was found to be safe and feasible. However, no appreciable gains in any of the primary ( $3.5 \mathrm{~m}$ vs $3 \mathrm{~m}$ ) or secondary outcomes variables were reported.The conceptual design of the trial focused on addition of interventional measures for the care of children with CP, incorporating a familybased model, which would be economically viable and feasible in resource-limited settings. The authors recognized the older ages of children selected for the study as a confounding factor, given more limited adaptive responses later during childhood, to the positive effects of earlier developmental neuroplasticity.

A recent systematic review specifically discussed the feasibility and effectiveness of home-based physiotherapy programs in children with cerebral palsy, and found large variability in the study design, patient selection, intervention characteristics, and outcome measures [2]. Overall compliance to home-based training program implementation was moderate to high, ranging from $56-99 \%$ and an improvement in arm-hand performance within group across time was demonstrated [2]. Parent interviews highlight the key role of parent/family coaching and partnership with interdisciplinary care teams for continued motivation and success of home-based rehabilitative care [3].

Indian Pediatrics
The concept of 'First 1000 days' stresses the greater potential for positive adaptive effects of developmental neuroplasticity to interventions during critical/sensitive periods of brain development [4]. This concept includes timelier diagnoses for $\mathrm{CP}$-risk children during prenatal trimester-specific, neonatal and early childhood timeperiods [5]. Outcome studies of therapeutic interventions for children with $\mathrm{CP}$ require examinations of younger children before two years of age. During this time-period, rehabilitative care and therapies in a child's home can leverage the more positive adaptive effects of consistent parental involvement. Establishing high compliance by families to maintain their daily environmental routines, with continued supervision and training with a trained team of health professionals empowers parents to develop a strong partnership for long-standing success.

The COVID-19 pandemic reinforces global social, economic, psychological, educational and healthcare challenges for children with $\mathrm{CP}$ and their families. These confounding factors are specific to resource-rich or poor nations, including 'medical deserts' within countries where medical care is potentially more available. Innovative technologies such as improved telemedicine combined with expanded outreach services across all therapy-based developmental domains can potentially improve opportunities for more effective interventions. Ben-Pazi, et al. [6] emphasized more timely and consistent digital healthcare (telemedicine) for children with $\mathrm{CP}$ to encourage high levels of participation, with families as stakeholders motivated to the success of therapy programs. Community-based telemedicine kiosks within resourcepoor communities would utilize publicly owned computers, smartphones, or software provided by this program for virtual initial and follow-up consultations with multidisciplinary care teams. 
Future feasibility studies would assess strategies of video-monitoring of home-based therapies with greater supervision and parental training to achieve more positive results. Feasibility studies require larger sample sizes with younger age groups to assess the beneficial effects of environmental interventions during the first 1000 days. India is the second largest user of mobile phones worldwide, relying on this technology to provide health information and supportive healthcare in rural Indian regions [7]. Future studies that incorporate digital health technology such as telemedicine would expand the interventional tools and access platforms needed to reduce neurologic morbidities.

King, et al. [8] stressed the conceptual framework of family-centered care for service delivery as the best practice for early intervention of pediatric rehabilitative care. Family-centered service recognizes the unique nature of family as the key factor for the continuity of care to address a child's abilities and needs [8]. Strengths and deficiencies of each child-family unit are taken into consideration, to formulate the most effective team, resource, therapy and community support to optimize favorable outcome for a child with $\mathrm{CP}$. This experimental design concept was presented by Goswami, et al. [1] as an important contribution to guide the direction of future research with a family-centered approach.

Outcomes research of rehabilitative care for children with $\mathrm{CP}$ is presently limited by the heterogeneity of selected patient populations and study designs, and by paucity of instruments and scales to accurately measure potentially subtle but important functional improvement. Given that the challenges of effective rehabilitative care, community integration, accessibility, resources and outcomes research vary worldwide, countries with large and diverse populations like India could conduct larger trials with greater statistical power analyses. Integration of digital technology platforms, home-based care with a family-centered approach that serve children with $\mathrm{CP}$ as well as other neurodevelopmental disorders could be more critically investigated to improve patient-centric and population-based care practices. Global initiatives and partnerships need to support all nations and regions with funding and resources challenges. This will contribute to the worldwide reduction in the burden from brain disorders across the life span [4].

Contributors: SA: reviewed the literature and the paper for the editorial, prepared the manuscript, reviewed edits, and finalized the final version; MS: reviewed the paper and the editorial, edited, and critically reviewed the final version; AT: reviewed the paper and the editorial, edited, and critically reviewed the final version.

Funding: None; Competing interests: None stated.

\section{REFERENCES}

1. Goswami JN, Sankhyan N, Singhi, P. Add-on home-centered activity-based therapy vs conventional physiotherapy in improving walking ability at 6-months in children with diplegic cerebral palsy: A randomized controlled trial. Indian Pediatr. 2021;58:826-32.

2. Becker LW, Schnackers ML, Janssen-Potten YJ, Kleijnen J, Steenbergen B. Feasibility and effect of home-based therapy programs for children with cerebral palsy: a protocol for a systematic review.BMJ Open. 2017;7:e013687.

3. Novak I. Parent experience of implementing effective home programs. Phys Occup Ther Pediatr 2011;31:198-213.

4. Scher MS. "The First Thousand Days" define a fetal/neonatal neurology program. 2021. Front Pediatr. 2021;9:612.

5. Scher MS. Fetal/neonatal neurology training for neurologists. Neurology. 2020;95:947-48.

6. Ben-Pazi H, Beni-Adani L, Lamdan R. Accelerating telemedicine for cerebral palsy during the COVID-19 pandemic and beyond. Frontiers Neurol. 2020;11:746.

7. Sreejith D, Menon V. Mobile phones as a medium of mental health care service delivery: perspectives and barriers among patients with severe mental illness. Indian J Psychol Med. 2019;41:428-33.

8. King S, Teplicky R, King G, Rosenbaum P. Family-centered service for children with cerebral palsy and their families: a review of the literature. Semin Pediatr Neurol. 2004;11: 78-86. 